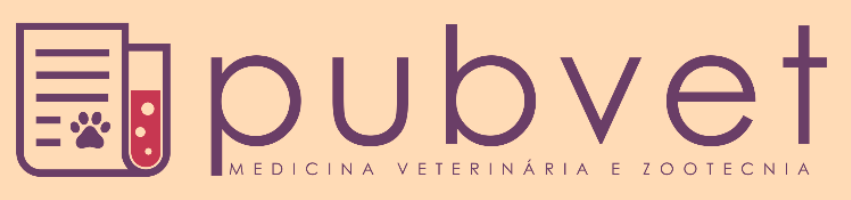

https://doi.org/10.31533/pubvet.v15n09a924.1-8

\title{
Carcinoma cribriforme mamário em paciente felina: Relato de caso
}

\author{
Niciérgi Pereira Medeiros de Menine ${ }^{1} * \bullet$, Géssica Gissele Almeida da Silva Araújo ${ }^{2} \bullet$, Marcelo \\ de Lima Wulff ${ }^{\circ}$
}

'Professora do curso de medicina veterinária do Centro Universitário Ritter dos Reis, UniRitter. Porto Alegre -RS Brasil. ${ }^{2}$ Doutoranda do Programa de Pós-Graduação da Universidade Federal Rural de Pernambuco. Pernambuco-PE. Brasil. ${ }^{3}$ Médico veterinário especialista em diagnóstico por imagem/ultrassonografia em pequenos animais.

*Autor para correspondência, E-mail: niciergi.menine@uniritter.edu.br

\begin{abstract}
Resumo. A incidência dos tumores mamários malignos na espécie felina é alta, sendo os carcinomas os mais frequentes. O carcinoma cribriforme mamário é neoplasia maligna, caracterizada pela agressividade na espécie, apesar disso, existe escassez acerca do tema e informações discrepantes em alguns tópicos. Revelando importância de publicações sobre o assunto para melhoria na qualidade do atendimento e tratamento instituído pelo médico veterinário oncologista. $\mathrm{O}$ presente estudo objetivou relatar o caso de uma paciente felina, castrada tardiamente, diagnosticada com carcinoma mamário cribriforme. Ao decorrer do tratamento, mesmo tendo realizado procedimento cirúrgico de mastectomia radical bilateral e instituição de protocolo quimioterápico adjuvante juntamente com inibidor de cicloxigenases, conforme indicado em literatura consultada, a paciente apresentou recidiva tumoral local, sendo avaliada como inoperável. Conclui-se então que o prognóstico é ruim, independente do protocolo de tratamento instituído, pois ocorrem, quase que invariavelmente, recidivas locais e metástases à distância.
\end{abstract}

Palavras-chave: Mastectomia, oncologia, quimioterapia

\section{Mammary cribriform carcinoma in a feline patient: Case report}

Abstract. The incidence of malignant mammary tumors in the feline species is high, with carcinomas being the most frequent. Cribriform mammary carcinoma is a malignant neoplasm, characterized by aggressiveness in the species, despite this, there is a shortage on the subject and discrepant information on some topics. Revealing the importance of publications on the subject for improving the quality of care and treatment instituted by the veterinary oncologist. The present study aimed to report the case of a feline patient, castrated late, diagnosed with cribriform breast carcinoma. During the course of her treatment, despite having undergone a bilateral radical mastectomy surgical procedure and the institution of an adjuvant chemotherapy protocol together with a cyclooxygenase inhibitor, as indicated in the consulted literature, the patient presented local tumor recurrence, being assessed as inoperable. It is concluded that the prognosis is poor, regardless of the treatment protocol established, as local recurrences and distant metastases occur almost invariably.

Keywords: Chemotherapy, mastectomy, oncology

\section{Introdução}

As neoplasias são consideradas entre a segunda e terceira causa de óbito na espécie felina (Batista et al., 2016; Manteigas et al., 2013). Sendo os neoplasmas mamários o segundo mais frequente (Barboza et al., 2019; Togni et al., 2013) e terceiro em incidência para De Nardi et al., (2017 ). A precocidade do diagnóstico tem efeito fundamental no prognóstico e tratamento das neoplasias (Corrêa 
et al., 2016). Animais velhos (média idade 10 a 12 anos) e inteiros são usualmente afetados (Morris \& Dobson, 2007). Dentre os tumores malignos mamários, os carcinomas são os mais prevalentes, seguidos dos carcinossarcomas (Togni et al., 2013,2018).

O tratamento de escolha para neoplasias mamárias em felinos é a mastectomia radical (Campos et al., 2016; Morris, 2013) com remoção de linfonodos inguinais e axilares, sendo o mais eficiente no controle de recidivas (Morris, 2013). Associado ao tratamento cirúrgico, o emprego de citostáticos para tumores de mama também se mostram efetivos (Cirillo, 2008).

Os carcinomas cribriformes, que são objeto desse relato, possuem prognóstico reservado, uma vez que são neoplasmas malignos de reconhecido potencial invasivo e metastático (Oliveira et al., 2020). Os tumores mamários em felinos ainda são pouco estudados em relação aos tumores mamários caninos, isso reforça a importância dos estudos nessa espécie (Oliveira et al., 2020).

O presente trabalho tem como objetivo reportar caso de uma paciente felina acometida por carcinoma mamário cribriforme, enfatizando sua agressividade, capacidade de promover recidivas locais e metástases à distância, independente de protocolos terapêuticos instituídos. Acerca disso, encontra-se uma escassez em literatura sobre tratamento específico em gatas, o que difere dos diversos relatos encontrados em cadelas. E revela a importância de publicações sobre o assunto para melhoria na qualidade do atendimento e tratamento instituído pelo médico veterinário oncologista.

\section{Material e métodos}

Atendida paciente felina, fêmea, nove anos de idade, sem raça definida, vacina quíntupla em dia, negativa para o teste que detecta doenças virais como imunodeficiência felina e leucemia viral felina (Fiv/Felv), domiciliada, contactantes hígidos, procedimento de ovariohisterectomia eletiva realizado aos quatro anos de idade conforme relato de tutores. Durante anamnese, foi referida cirurgia de mastectomia unilateral esquerda três meses antes do atendimento.

Ao exame clínico minucioso foi constatado nódulo em M4 direita, pequeno, aproximadamente 0,5 $\mathrm{cm}$, com consistência firme à palpação, superfície regular, aderido, linfonodo inguinal ipisilateral levemente aumentado na palpação, demais parâmetro sem alterações, paciente normocorada, normorexia, normúria, normoquesia, temperatura retal em $37,8^{\circ} \mathrm{C}$ e ausculta de campo cardiopulmonar sem alterações evidentes. Coletado amostra de sangue para hematologia e bioquímica básica, devido restrições de custos. No momento da consulta solicitado exames de imagens complementares para correto estadiamento do paciente oncológico, inicialmente solicitado a radiografia torácica em três projeções (ventrodorsal, lateral direita e lateral esquerda) e ecografia abdominal total. Exame de citologia aspirativa por agulha fina (CAAF) de linfonodo inguinal reativo, para classificar status nodal regional, pré-cirurgia, não autorizado devido aos custos.

Paciente prontamente encaminhada para exames pré-operatórios e novo procedimento cirúrgico de mastectomia unilateral direita e amostra biológica enviada para análise histopatológica juntamente com avaliação de margens cirúrgicas. Permaneceu internada no pós-operatório e alta se processou no terceiro dia. Solicitado retorno em 10 dias para retirada de pontos.

\section{Resultados e discussão}

O primeiro laudo de histopatológico, da cadeia mamária esquerda, apresentou diagnóstico morfológico de carcinoma cribriforme grau II, sendo neoplasia maligna com linfonodo inguinal livre de células neoplásicas. Laudo corrobora com os estudos preliminares de Millanta et al. (2006) que mostram o comportamento maligno dos tumores mamários na espécie felina.

Diferentemente do descrito por Morris \& Dobson (2007), onde pacientes com neoplasias mamárias apresentam idade média de ocorrência entre 10 e 12 anos, a paciente em questão, no momento do diagnóstico estava com nove anos de idade. A paciente foi castrada tardiamente, corroborando assim, com Daleck et al. (2016), onde os mesmos descreveram a redução significativa do risco de desenvolvimento em tumores mamários em animais precocemente submetidos a procedimento de ovariohisterectomia. 
Após exame clínico constatar presença de nódulo em mama, a paciente foi encaminhada para realização de exames hematológicos e bioquímicos básicos (creatinina e ALT) pré-operatórios, ambos com resultados dentro dos parâmetros. Deve-se considerar que a creatinina de forma isolada é considerada um marcador de injúria renal tardio (Freitas et al., 2014).

Exames complementares de imagem como ultrassonografia abdominal e radiografia de tórax em três projeções foram realizadas e não encontradas metástases à distância, considerando as limitações de identificação dos tipos de estudos empregados. Solicitado a Tomografia Computadorizada, ressaltando o fato que as radiografias torácicas não conseguem identificar a presença de metástase de nódulos menores de sete milímetros conforme estudo retrospectivo de Nemanic et al. (2006), mas não autorizado devido aos custos.

O ecocardiograma também não foi autorizado pelo mesmo motivo supracitado e exame de urina não realizada pelo fato da paciente ser pouco colaborativa, em diversas ocasiões procedeu-se tentativas de coleta por compressão vesical ou por cistocentese guiada pela ultrassonografia, com paciente sempre anestesiada e devidamente monitorada, mas sem sucesso nessas tentativas. O Guideline da International Renal Interest Society (IRIS, 2019) sugere associação de exames no paciente não azotêmico, como o exame de urina, para avaliação da habilidade dos rins em concentrar a mesma, identificação de proteinúria renal e avaliação da relação proteína/creatinina urinária, objetivando a identificação de lesões renais precoces. Essas associações de exames visam auxiliar na prevenção da doença renal e retardar sua progressão (Waki et al., 2010).

Após concluídos exames pré-operatórios, paciente encaminhada prontamente para procedimento cirúrgico assim com indicado pelos autores Estralioto \& Conti (2019). A mastectomia bilateral ocorreu em duas etapas (12 semanas entre procedimentos), o intervalo entre elas foi superior ao indicado de quatro a seis semanas por Papazoglou et al. (2014), devido à resistência dos tutores a mais uma intervenção cirúrgica.

Os medicamentos administrados em internamento no pós-operatório foram metadona $(0,1 \mathrm{mg} / \mathrm{kg}$ QID durante 2 dias), dipirona (25mg/kg IV SID durante 3 dias), meloxicam (0,05 mg/kg SID IV durante 3 dias) e amoxicilina com clavulanato de potássio (12,5 mg/kg VO BID durante 3 dias), este último, os tutores deram sequência em domicílio até completar 7 dias.

O pós-operatório mostrou-se complicado, paciente não permitindo manipulação, demonstrou sinais de dor, como agressividade, anorexia e muito relutante em realizar movimentações até mesmo para urinar e defecar. A dor é de difícil reconhecimento nas espécies, mas pode alterar diversos sistemas, e levar a retenção urinária conforme Aleixo et al. (2017). O mesmo foi observado na paciente durante período de internamento. Em estudo realizado por Horta (2013), em cadelas, mostrou alterações fisiológicas bem importantes em pós-operatório de mastectomia, ressaltando a agressividade do procedimento. A alta ocorreu após três dias internada, onde já apresentava sinais clínicos estáveis assim como comportamento normalizado.

Retornou para retirada de pontos com dez dias de pós-operatório, não ocorreu deiscência ou intercorrências na região, no mesmo período o laudo do exame histopatológico foi liberado pelo laboratório. Laudo constatou mesmo tipo histológico tumoral e mesma graduação da primeira amostra (carcinoma cribriforme grau II), linfonodo de região inguinal, mais uma vez, ausente de células neoplásicas e margens com classificação em M3 (células neoplásicas a 2-5 mm das margens cirúrgicas), apresentando contagem mitótica de 50.

Essa recidiva tumoral pode estar ligada com as drenagens linfáticas contralaterais já citadas em estudo realizado por Pereira et al. (2003), côngruo com esses autores, as drenagens linfáticas ocorrem de maneira ipisilateral e contralateral. Essas ligações acontecem também animais sadios e são mais evidentes em animais com neoplasias mamárias. Na medicina humana existem diversos estudos sobre os mecanismos da linfangiogênese (formação de novos vasos linfáticos) e, conforme Andrade (2008), a sua elucidação pode beneficiar pacientes oncológicos, pelos bloqueios desse mecanismo, assim prevenindo metástases linfáticas.

Outro estudo realizado por Pereira et al. (2013) fazendo uso de técnicas de linfocintilografia para identificação de drenagem linfática das glândulas mamárias em cadelas, demonstrou a existência de 
comunicação entre linfonodos superficiais e profundos, assim a remoção cirúrgica apenas de linfonodos axilares e inguinais para controle de recidivas sugerido por Morris (2013) não seria efetivo. Consequentemente, mesmo com o laudo da paciente deste relato apresentando ambos linfonodos inguinais livres de metástases, não é possível excluir então que a mesma possa ocorrer, pois não foram investigados linfonodos axilares, uma vez que nódulos estavam presentem em M4 e linfonodos inguinais seriam os drenantes da região, assim como não foram avaliados os mais profundos para determinar a ausência de células neoplásicas. Contudo, não foram encontrados relatos semelhantes para espécie felina. Apesar da via linfática ser a principal para disseminação de metástases de carcinomas, a via hematogênica também é importante devido permeação de células em capilares e vênulas segundo Zuccari et al. (2001), ou seja, mesmo que se procedesse a linfadenectomia, tanto de linfonodos superficiais e profundos com auxílio de contrastes, a via hematogênica também poderia ser causadora dessa recidiva tumoral. A realização de imuno-histoquímica, traria informações tanto prognósticas como preditivas, de extrema importância (Daleck et al., 2016; Ribeiro et al., 2009; Silva, 2015), mas devido alto custo no somatório do tratamento, tutores optaram por não realizar.

Indicado quimioterapia adjuvante seguindo as indicações de literatura (Cirillo, 2008; Daleck et al., 2016; McNeill et al., 2009). Optou-se por não utilizar o protocolo de escolha com doxorrubicina como o sugerido por Cirillo (2008), devido ao risco de lesão renal irreversível e pelo fato de realizar apenas a creatinina como marcador de função renal sem os demais exames indicados pela (IRIS, 2019). Utilizou-se a carboplatina que têm se mostrado segura conforme descrito por Kisseberth et al. (2008), sendo bem tolerado pelos pacientes com mínimos efeitos colaterais observados (Campos et al., 2014) e efeitos colaterais estão mais relacionados aos gastrointestinais segundo Silveira (2016), o mesmo não foi observado durante todo tratamento quimioterápico da paciente em questão.

Indicado carboplatina na dose de $200 \mathrm{mg} / \mathrm{m}^{2} \mathrm{IV}$ a cada 21 ou 28 dias, uma vez que Kisseberth et al. (2008) referem que este citostático é seguro quando administrado nesses intervalos de tempo e na dose até de $240 \mathrm{mg} / \mathrm{m}^{2}$. Preconizado quatro sessões ao total, estando dentro do descrito por Daleck et al. (2016) que indicam entre três a seis sessões. Empiricamente, foi associado ao protocolo, o uso de piroxicam, uma vez que, uma alta expressão de COX-2, em felinos com carcinomas, foi detectada em estudo realizado por Millanta et al. (2006). Campos et al. (2016) mostraram que sua expressão participa do processo da formação do câncer (carcinogênese). Inibidores de COX-2 seletivos são mais indicados por possuírem menores efeitos deletérios que os não seletivos (Monteiro et al., 2019). Neste caso, julgou-se adequado o uso do piroxicam (inibidor não seletivo de cox-2) em detrimento de um inibidor seletivo de cox-2, devido seu baixo custo e facilidade de administrar o fármaco a cada 48 horas. Fato que contribuiu para adesão ao tratamento pelos tutores devido paciente não ser colaborativa durante manipulação. A paciente não apresentou nenhum efeito colateral durante seu uso na dose de $0,3 \mathrm{mg} / \mathrm{kg}$ a cada 48 horas por via oral., consolidando dados de Bulman-Fleming et al. (2010). Previamente a cada sessão, foram realizados exames hematológicos e bioquímicos, todos sem alterações significativas.

As três primeiras sessões ocorreram no intervalo exato de 21 dias e entre terceira e quarta sessão, por questões relacionadas aos tutores, intervalo foi de 23 dias. Administrado previamente ao citostático, a difenidramina (2 $\mathrm{mg} / \mathrm{kg}$ IV) classificado com antiemético e anti-histamínico para prevenção de reações adversas acordante com indicado por Daleck et al. (2016), onde os mesmos indicam a aplicação deste fármaco, 15 a 20 minutos antes de cada sessão de quimioterapia, para evitar reações de hipersensibildade. Todas as sessões ocorreram com animal sedado, pois paciente não colaborativo, o mesmo foi devidamente acompanhado pelo anestesista.

Clinicamente a paciente se manteve estável, normorética, sendo acompanhada em média a cada 2-3 meses. Novas radiografias da cavidade torácica à procura de metástase à distância foram realizadas cinco meses após segundo procedimento cirúrgico (Figura 1).

Laudos sem presença de metástases visíveis para o tipo de técnica empregada. Sendo novamente reforçado com os tutores a necessidade da realização de tomografia computadorizada para visualização de metástases e novamente tutores não autorizaram a realização do exame pelos custos.

Em nova avaliação, oito meses após segunda cirurgia e quatro meses após término de protocolo quimioterápico, apresentou diversos nódulos em abdome, região de tecido subcutâneo, mas bem 
aderidos em musculatura, localizados em região inguinal esquerda, de grande extensão e de forma disseminada. Realizada citologia aspirativa por agulha fina (CAAF), com laudo compatível com a suspeita de recidiva de carcinoma.

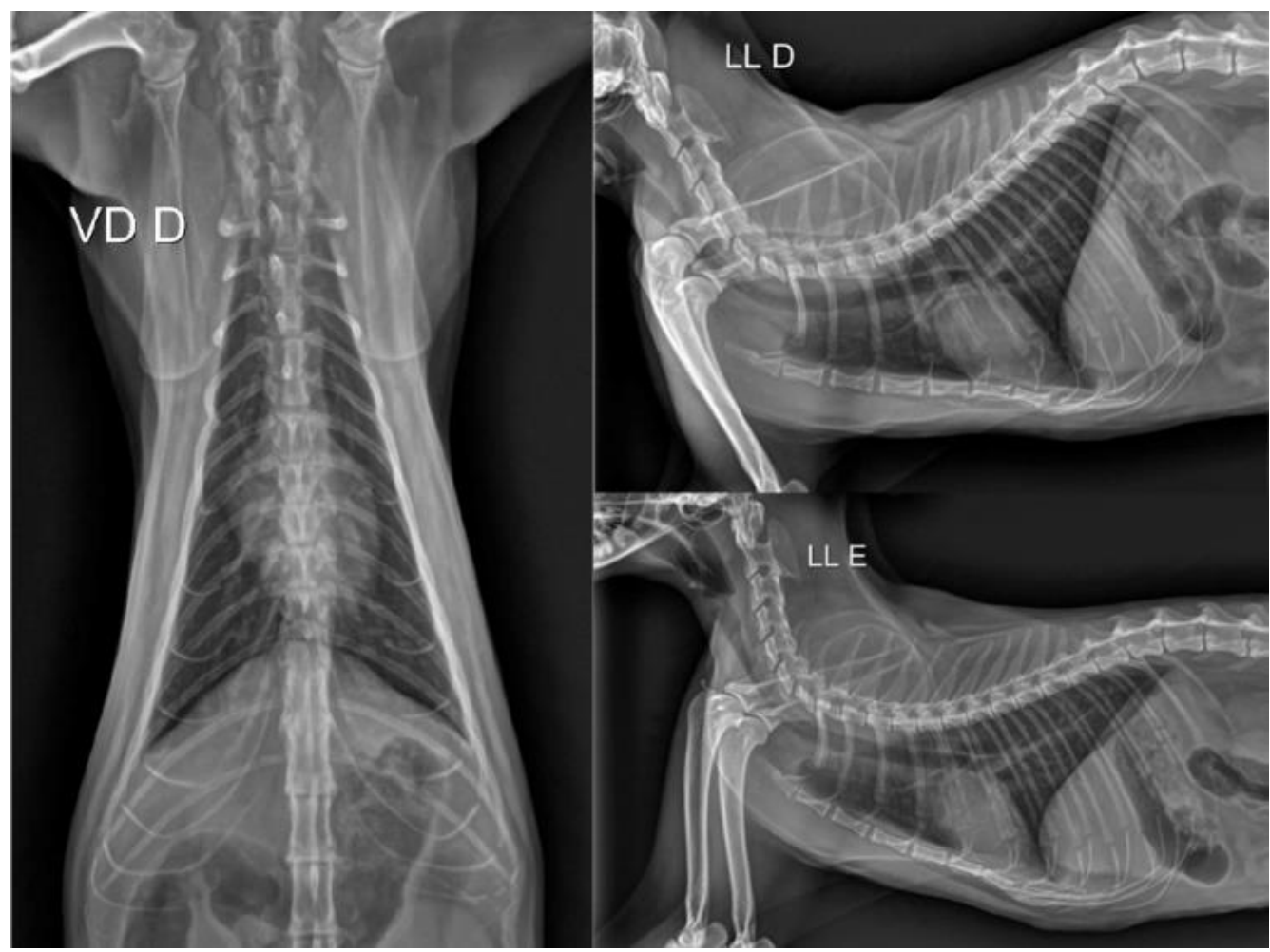

Figura 1. imagem do exame radiográfico projeções onde A) Ventrodorsal B) Lateral direita c) Lateral esquerda para pesquisa de metástase a distância em cavidade torácica. Imagens gentilmente cedidas por M.V. Esp Marília Correa Borba.

Paciente foi encaminhada novamente para avaliação com médico veterinário cirurgião, onde não foi indicado o procedimento cirúrgico devido grau de disseminação desses nódulos. No intuito de retardar a progressão da doença ou sintomatologia associada ao câncer, indicado a quimioterapia de forma paliativa. A indicação é com base em extrapolações da Medicina Humana, onde as Diretrizes Diagnósticas e Terapêuticas do Carcinoma de Mama, publicado pelo Ministério da Saúde (BRASIL, 2018), mostram essa possibilidade de aplicação da quimioterapia para casos redicivantes e inoperáveis como da paciente em questão.

Corroborando com todos os estudos avaliados para este relato, a grande agressividade dos tumores mamários na espécie felina independente do protocolo de tratamento instituído. O tamanho tumoral mostrou seguir os parâmetros de mau prognóstico, tanto em relação a sobrevida global como o tempo livre de doença conforme descrito por Daleck et al. (2016). Uma vez que ao diagnóstico, a paciente apresentava, o nódulo primário em M4 esquerda de 1,6 X 7,1 cm e apresentou apenas nove meses de tempo livre de doença. Diferentemente do relatado por estes mesmos autores, onde existem estudos que demonstram o intervalo entre diagnóstico e óbito do paciente de 10 a 12 meses, a paciente em questão apresentou intervalo de 15 meses. A gradação histológica II e presença de muitas figuras de mitose apresentaram mau prognóstico assim como afirmação de Schirato et al. (2012) e também confirmado em estudo realizado por Jorge et al. (2018) onde a gradação histológica demonstrou melhor representatividade sobre potencial infiltrativo e metastático.

\section{Conclusões}

Torna-se claro a agressividade do carcinoma cribriforme mamário em pacientes felinos, pois apesar de serem observadas respostas variáveis conforme o indivíduo, é perceptível que independente do protocolo de tratamento instituído, ocorre a recidiva local ou metástase à distância, seja por via linfática ou hematogênica. Outro ponto a ser considerado é adesão dos tutores ao tratamento, seja por 
questões financeiras, crenças, ou dificuldade no manejo do paciente em casa. Há ainda necessidade de estudos específicos na espécie para determinar melhor os valores prognósticos e preditivos.

\section{Referências bibliográficas}

Aleixo, G. A. S., Tudury, E. A., Coelho, M. C. O. C., Andrade, L. S. S., \& Bessa, A. L. N. G. (2017). Tratamento da dor em pequenos animais: classificação, indicações e vias de administração dos analgésicos (revisão de literatura: parte II). Medicina Veterinária, 11(1), 29-40. DOI: https://doi.org/10.26605/medvet-n1-1596

Andrade, M. (2008). Linfangiogênese e genética dos linfedemas: revisão da literatura. Jornal Vascular Brasileiro, 7, 256-261. DOI: https://doi.org/10.1590/s1677-54492008000300011

Barboza, D. V., Grala, C. X., Silva, E. C., Salame, J. P., Bernardi, A., Silva, C. B., \& Guim, T. N. (2019). Estudo retrospectivo de neoplasmas em animais de companhia atendidos no hospital de clínicas veterinárias da universidade federal de Pelotas durante 2013 a 2017. PUBVET, 13, 152. DOI: https://doi.org/10.31533/pubvet.v13n4a312.1-12

Batista, E. K. F., Pires, L. V., Miranda, D. F. H., Albuquerque, W. R., Carvalho, A. R. M., Santos Silva, L., \& Silva, S. M. M. S. (2016). Estudo retrospectivo de diagnósticos post-mortem de cães e gatos necropsiados no Setor de Patologia Animal da Universidade Federal do Piauí, Brasil de 2009 a 2014. Brazilian Journal of Veterinary Research and Animal Science, 53(1), 88-96. DOI: https://doi.org/10.11606/issn.1678-4456.v53i1p88-96

Bulman-Fleming, J. C., Turner, T. R., \& Rosenberg, M. P. (2010). Evaluation of adverse events in cats receiving long-term piroxicam therapy for various neoplasms. Journal of Feline Medicine and Surgery, 12(4), 262-268. DOI: https://doi.org/10.1016/j.jfms.2009.09.007

Campos, Cecilia B, Damasceno, K. A., Gamba, C. O., Ribeiro, A. M., Machado, C. J., Lavalle, G. E., \& Cassali, G. D. (2016). Evaluation of prognostic factors and survival rates in malignant feline mammary gland neoplasms. Journal of Feline Medicine and Surgery, 18(12), 1003-1012. DOI: https://doi.org/10.1177/1098612x15610367

Campos, Cecilia Bonolo, Nunes, F. C., Lavalle, G. E., \& Cassali, G. D. (2014). Use of surgery and carboplatin in feline malignant mammary gland neoplasms with advanced clinical staging. In Vivo, $28(5), 863-866$.

Cirillo, J. V. (2008). Tratamento quimioterápico das neoplasias mamárias em cadelas e gatas Chemotherapeutic treatment of mammary neoplasms in female dogs and cats. Revista Do Instituto de Ciências Da Saúde, 26(3), 325-327.

Corrêa, L. G., Zamboni, R., Scheid, H. V., Brunner, C. B., \& Sallis, E. S. V. (2016). Estudo retrospectivo de tumores em felinos na região sul do Rio Grande do Sul. XXV Congresso de Iniciação Científica Da Universidade Federal de Pelotas.

Daleck, C. R., Fonseca, C. S., \& Canola, J. C. (2016). Oncologia em cães e gatos. Roca.

De Nardi, A. B., Ferreira, T. M. M. R., Assunção, K. A. (2017). Neoplasias Mamárias. In: Daleck, C. R., De Nardi, A. B. Oncologia em Cães e Gatos. 2.ed. Rio de Janeiro: Roca., Cap 40 499- 516.

Estralioto, B. L., \& Conti, J. (2019). Câncer de mama em cadelas - atualidades do diagnóstico e prognóstico ao tratamento cirúrgico. Enciclopédia Biosfera, 16(29), 444-463. DOI: https://doi.org/10.18677/encibio_2019a33

Freitas, G. C., Veado, J. C. C., \& Carregaro, A. B. (2014). Testes de avaliação de injúria renal precoce em cães e gatos. Semina: Ciências Agrárias, 35(1), 411-426. DOI: https://doi.org/10.5433/1679$\underline{0359.2014 \mathrm{v} 35 \mathrm{n} 1 \mathrm{p} 411}$

Jorge, M. F., Tomacheuski, R. M., Hataka, A., \& Sequeira, J. L. (2018). Abordagem da proliferação celular pela técnica de imuno-histoquímica com biomarcador ki-67 e caracterização histopatológica no carcinoma mamário felino. Revista Unimar Ciências, 27(1-2).

Horta, R. S. Avaliação da nocicepção, complicações pós-operatórias e impacto na qualidade de vida provocados por duas técnicas cirúrgicas de mastectomia na espécie canina. 2013. 95 f. Dissertação (Medicina Veterinária). Universidade Federal de Minas Gerais. Belo Horizonte - BH. 2013 
International Renal Interest Society - Staging of Chronic Kidney disease (modified 2019). Disponível http <://www.iris-kidney.com/guidelines/staging.html > Acesso em 03 jun 2020.

Kisseberth, W. C., Vail, D. M., Yaissle, J., Jeglum, K. A., Couto, C. G., Ward, H., Khanna, C., \& Obradovich, J. E. (2008). Phase I clinical evaluation of Carboplatin in tumor-bearing cats: a veterinary cooperative oncology group study. Journal of Veterinary Internal Medicine, 22(1), 8388. DOI: https://doi.org/10.1111/j.1939-1676.2007.0017.x

Manteigas, F. M. S., Godinho, A., \& Almeida, A. (2013). Causas de mortalidade em gatos com mais de nove anos: Estudo retrospetivo de cem casos. Revista Lusófona de Ciência e Medicina Veterinária, 6, 47-57.

McNeill, C. J., Sorenmo, K. U., Shofer, F. S., Gibeon, L., Durham, A. C., Barber, L. G., Baez, J. L., \& Overley, B. (2009). Evaluation of adjuvant doxorubicin-based chemotherapy for the treatment of feline mammary carcinoma. Journal of Veterinary Internal Medicine, 23(1), 123-129. DOI: https://doi.org/10.1111/j.1939-1676.2008.0244.X

Millanta, F., Citi, S., Della Santa, D., Porciani, M., \& Poli, A. (2006). COX-2 expression in canine and feline invasive mammary carcinomas: correlation with clinicopathological features and prognostic fmolecular markers. Breast Cancer Research and Treatment, 98(1), 115-120. DOI: https://doi.org/10.1007/s10549-005-9138-z

BRASIL. Ministério da Saúde. (2018). Diretrizes Diagnósticas e Terapêuticas do Carcinoma de Mama.Comissão Nacional de Incorporação de Tecnologias do SUS. Disponível em: < http://conitec.gov.br/images/Relatorios/2018/Relatorio_DDT_CAMama.pdf> Acesso em 03 jun 2020.

Monteiro, J., Monteiro, R., Okuyama, C., \& Diniz, S. (2019). importância da analgesia e do uso de anti-inflamatórios não esteroidais cox2 seletivos na prevenção e tratamento do câncer em cães. Enciclopédia Biosfera, 16(29), 2-9. DOI: https://doi.org/10.18677/encibio_2019a3

Morris, J, \& Dobson, J. (2007). Trato urinário. In J. Morris \& J. Dobson (Eds.), Oncologia em pequenos animais (pp. 155-165). Roca, Brasil.

Morris, Joanna. (2013). Mammary tumours in the cat: size matters, so early intervention saves lives. Journal of Feline Medicine and Surgery, 15(5), 391-400. DOI: https://doi.org/10.1177/1098612x13483237

Nemanic, S., London, C. A., \& Wisner, E. R. (2006). Comparison of thoracic radiographs and single breath-hold helical CT for detection of pulmonary nodules in dogs with metastatic neoplasia. Journal of Veterinary Internal Medicine, 20(3), 508-515. DOI: https://doi.org/10.1111/j.19391676.2006.tb02889.x

Oliveira, F. N. L., Soares, Y. G. S., Ferreira, M. A. S., Ramos, F. R., Oliveira, T. S., Carneiro, R. S., Galiza, G. J. N., \& Dantas, A. F. M. (2020). Carcinoma cribriforme metastático em uma gata. Revista de Agroecologia No Semiárido, 4(4), 82-86. DOI: https://doi.org/10.35512/ras.v4i4.4586

Papazoglou, L. G., Basdani, E., Rabidi, S., Patsikas, M. N., \& Karayiannopoulou, M. (2014). Current surgical options for mammary tumor removal in dogs. Journal of Veterinary Science \& Medicine, 2(1), 2-7. DOI: $10.13188 / 2325-4645.1000007$

Pereira, C. T., Rahal, S. C., Balieiro, J. C. C., \& Ribeiro, A. (2003). Lymphatic drainage on healthy and neoplasic mammary glands in female dogs: can it really be altered? Journal of Veterinary Medicine Series C: Anatomia Histologia Embryologia, 32(5), 282-290. DOI: https://doi.org/10.1046/j.1439-0264.2003.00485.x

Ribeiro, L., Damasceno, K. A., Costa Neto, J. M., D’Assis, M. J. M. H., Costa, A., Silva, N. dos S., Aguiar, P., Cassali, G. D., \& Lima, A. E. S. (2009). Expressão da COX-2 nos carcinomas mamários de cadelas. Revista Brasileira de Ciências Veterinárias, 13(4), 1-12.

Schirato, G. V., Silva, V. R., Menezes, R. C., Ribeiro, E. L., Peixoto, C. A., Neves, C. O., MeloJúnior, M. R., \& Porto, A. L. F. (2012). Caracterização histopatológica de tumores mamários espontâneos de gatas (Felis catus) atendidas no Hospital Veterinário da UFRPE (Recife, Pernambuco, Brasil). Revista Brasileira de Ciência Veterinária, 19(3), 203-205. DOI: https://doi.org/10.4322/rbcv.2014.096 
Silva, T. L. R. C. (2015). Classificação molecular de tumores mamários felinos e sua relevância clínica. Universidade de Lisboa.

Silveira, P. S. D. (2016). Efeitos secundários da quimioterapia antineoplásica e seu impacto na qualidade de vida em cães e gatos com doença oncológica. In Faculdade de Medicina Veterinária: Vol. Master of. Universidade de Lisboa.

Togni, M., Curtis, A., Vargas, D. P., Kommers, G. D., Irigoyen, L. F., \& Fighera, R. A. (2018). Causas de morte e razões para eutanásia em gatos na Região Central do Rio Grande do Sul (1964-2013). Pesquisa Veterinária Brasileira, 38(4), 741-750. DOI: https://doi.org/10.1590/1678-5150-pvb-5075

Togni, M., Masuda, E. K., Kommers, G. D., Fighera, R. A., \& Irigoyen, L. F. (2013). Estudo retrospectivo de 207 casos de tumores mamários em gatas. Pesquisa Veterinária Brasileira, 33(3), 353-358. DOI: https://doi.org/10.1590/s0100-736x2013000300013

Waki, M. F., Ribas Martorelli, C., Erdmann Mosko, P., \& Kogika, M. M. (2010). Classificação em estágios da doença renal crônica em cães e gatos: abordagem clínica, laboratorial e terapêutica. Ciência Rural, 40(10), 1-9. DOI: https://doi.org/10.1590/s0103-84782010005000168

Zuccari, D., Santana, A. E., \& Rocha, N. S. (2001). Fisiopatologia da neoplasia mamária em cadelasrevisão. Clínica Veterinária, 32(2), 50-54.

Histórico do artigo:

Recebido: 11 de maio de 2021

Aprovado: 1 de julho de 2021 .
Licenciamento: Este artigo é publicado na modalidade Acesso Aberto sob a licença Creative Commons Atribuição 4.0 (CC-BY 4.0), a qual permite uso irrestrito, distribuição, reprodução em qualquer meio, desde que $\mathrm{o}$ autor $\mathrm{e}$ a fonte sejam devidamente creditados. 